\title{
Blockchain for LBG Maritime Energy Contracting and Value Chain Management: A Green Shipping Business Model for Seaports
}

\author{
Robert PHILIPP ${ }^{1,2 *}$ \\ ${ }^{1}$ Hochschule Wismar, University of Applied Sciences: Technology, Business and Design, Wismar Business \\ School, Philipp-Müller-Str. 14, 23966 Wismar, Germany \\ ${ }^{2}$ Tallinn University of Technology, School of Business and Governance: Department of Business \\ Administration, Ehitajate tee 5, 12616 Tallinn, Estonia
}

\begin{abstract}
To reduce emissions in the maritime transport sector, the International Maritime Organisation (IMO) follows a global clean shipping strategy. Among the different directives of IMO, currently especially the sulphur emission regulations pose challenges for the shipping industry. Related to this are the established Sulphur Emission Control Areas (SECAs) and the introduced global sulphur cap. To comply with the sulphur restrictions, according to the present technological state of the art, ship-owners have three options for their existing fleet: the installation of emission abatement technologies, the switch to low sulphur fuels, or retrofitting for the usage of alternative fuels. Regardless which option is favoured, most often selected solutions still depend on fossil fuels. The reasons for this can be traced back to the fact that supply of biofuels is not ensured in ports and generally seen as no profitable solution. This paper develops and examines an innovative business model with a special focus on liquefied biogas (LBG). The study bases on collected qualitative and quantitative data, which was used by applying the Business Model Canvas. The results will highlight that the business model bears the potential to promote LBG supply. Next to this, the research will show that blockchain and smart contracts are able to foster the implementation of the business model and optimisation of value chain operations. Lastly, economic advantages were highlighted within a case study that refers to the seaport Karlskrona in Sweden and the RoPax ferries from Stena Line that travel back and forth to Gdynia seaport in Poland.
\end{abstract}

Keywords - Business model innovation; digitalisation; emission reduction; global sulphur cap; LBG "Liquefied Biogas"; LBM "Liquefied Bio-Methane"; SECA "Sulphur Emission Control Area"; small and medium-sized seaports; smart contracts

\section{INTRODUCTION}

Seaports are the backbone of the transport network without the worldwide economy could not exist in its present form [1]. Concerning Europe, $74 \%$ of goods imported and exported, and $37 \%$ of domestic trade is handled by ports [2]. In 2017, about four billion tonnes of freight and 415 million passengers passed through the 1,200 European ports [3], [4]. Furthermore, approximately 3 million people are employed directly or indirectly in ports across the EU Member States [5].

* Corresponding author.

E-mail address: robert.philipp@hs-wismar.de 
According to the International Transport Forum Forecast of the OECD [6], waterborne transport worldwide will grow by $327 \%$ until 2050, producing $238 \%$ more $\mathrm{CO}_{2}$ emissions. In Europe, it is expected that freight volumes will increase until 2050 by $216 \%$ - with additional $174 \% \mathrm{CO}_{2}$ emissions. Therefore, it can be assumed that there will be an enormous shift in commodity transportation [7]. This will extremely affect maritime and inland ports. Particularly in the case of small ports, harsh environmental, competitive and operational pressure is expected. Small ports, especially in the Baltic Sea Region (BSR) which represents a flagship region in Europe [8], [9], suffer from out-dated infrastructure, missing smart specialization, inadequate investments, and the absence of new business models that could contribute to blue and green growth [10] - as it is requested in the European Commission's guidelines (e.g. [11]).

The situation of ports in the BSR is even more challenging, due to the issued directives from the International Maritime Organisation (IMO) [12]. To ensure a sustainable reduction of emissions from shipping, the IMO follows a long-term global clean shipping strategy [13]. Thereby, in particular the sulphur emission regulations pose challenges for the shipping industry - namely the established Sulphur Emission Control Areas (SECAs) that are enacted in the IMO MARPOL Annex 2, and the introduced global sulphur cap [14]. SECAs exist in the BSR, North Sea and English Channel as well as along the US American and Canadian coastlines [15]. Similar SECA directives for shipping are the Chinese regulation for coastal waters, which entered into force in 2016, and the EU directive 2005/33/EC [16]. According to the latest gradual adjustment, from 1 January 2015, in SECAs, fuel for ships is not allowed to have a sulphur concentration (\% w/w) above $0.1 \%$. Next to this, on 1 January 2020 , the renewed global sulphur cap entered into force, which stipulates to use fuel for ships with no more than $0.5 \%$ (i.e. worldwide, outside of SECAs) [14]. Thus, the ease of use of heavy fuel oil (HFO) do not comply with these emission restrictions any more. By taking into account the current technological state of the art, ship-owners have three options for their existing HFO fleet in order to comply with these sulphur restrictions: the installation of emission abatement technologies like scrubbers, the switch to expensive low sulphur fuels (inside SECAs: low sulphur marine gas oil - LSMGO, ultra-low sulphur fuel oil - ULSFO; outside SECAs: very low sulphur fuel oil - VLSFO), or retrofitting for the usage of alternative fuels (e.g. liquefied natural gas - LNG) [17]. Regardless, which option is favoured, selected solutions usually still depend on fossil fuels. Thereby, the majority of ship-owners, who primarily operate in SECAs, switched to LSMFO and ULSFO, which - compared to LNG are more expensive, but currently fulfil the requirements of the emission regulations and do not require comprehensive retrofits. This compliance option is also fostered by a general low oil price, since 2014 [18]. Moreover, another reason for low utilisation of biofuels that comply with these emission directives can be traced back to the fact that biofuel supply at competitive market prices is not ensured in ports and thus, often seen as too expensive solutions. In addition, supply of alternative fuels is associated with high investment costs for new infrastructure in seaports [16].

Further challenges, combined with additional investment needs arise from the digital transformation in the maritime industry [19], [20], which must be coped by small ports as well in order to remain competitive. According to Philipp et al. [10], [21], [22], small seaports in the BSR have a high interest in digitisation, but often do not have the necessary skills and capabilities as well as knowledge regarding the already existing wide range of ICT solutions (Information and Communications Technology) or current trends like Blockchain, Industry 4.0 and IoT (Internet of Things) - including the resulting potentials and opportunities. However, despite the lack of know-how, these framework conditions do not only pose risks, 
but can be seen as development opportunities for smaller ports, too. For example, digitisation in the maritime logistics sector bears the potential for the creation of new business models. Moreover, scientific studies have shown that especially in the context of Industry 4.0, small and medium-sized enterprises can take advantage (e.g. [23]), which on the other hand should also apply to small and medium-sized ports.

Against this background, the research objective of the present study is to develop and examine an innovative business model for regional ports with a special focus on liquefied biogas (LBG) in order to promote supply and utilisation of biofuels and renewable energy. Thereby, the present study refers among other things to the field-to-ferry concept from the GoLNG project and the Maritime Energy Contract (MEC) model, which was theoretically proposed for fuel producers by Olaniyi et al. [13], [24], [25]. These models will be adapted to regional seaports, which ensures a more realistic case, due to the fact that the port authorities and/or operators are - next to policy makers - the key players for the transition towards a sustainable biofuel supply and utilisation, as they are the decision makers for the needed bunkering infrastructure and suprastructure developments in the seaports. Furthermore, in the frame of an LBG value chain, traditional fuel producers are usually superfluous. The research bases on collected qualitative and quantitative data that was compiled by a desk research as well as expert interviews, and subsequently used in the frame of the applied Business Model Canvas from Osterwalder and Pigneur [26]. The study addresses the research question how the actions and transactions can be controlled between small and medium-sized ports and ship-owners as well as other potential stakeholders, plus which advantage and added value may be achieved through entrepreneurial collaboration between the key parties. The research results will showcase that the elaborated business model bears the potential to promote LBG supply, as well as that blockchain and smart contracts are enabler for optimised value chain operations and at the same time facilitate the implementation and functioning of the LBG MEC business model. Lastly, a comparative analysis with ULSFO was carried out in the frame of a case study in the medium-sized seaport Karlskrona (Sweden).

The paper is structure as follow: In the second chapter, the theoretical background is given, concerning the MEC and field-to-ferry concept, LBG as an emission compliance biofuel for ships, blockchain and smart contracts, as well as the Trans-European Transport Network (TEN-T) in order classify the European ports and to point out potential financial support schemes for infrastructure developments. Afterwards the used methodology is set out. Building upon this, the research results are showcased, whereby the article rounds up with a discussion and conclusion.

\section{THEORETICAL BACKGROUND}

\subsection{Trans-European Transport Network and Port Classification}

The European guidelines for the development of the TEN-T have identified 329 key ports that will become part of a unified network, which will boost growth and competitiveness in Europe's Single Market [27]. The TEN-T will be double-layered; on the one hand, it will consist of a comprehensive network and on the other hand of a core network. According to the corresponding European guidelines, the core network shall be created by 2030 and the comprehensive network by 2050 . Inside the core network, nine corridors are planned. These corridors will be multi-modal and shall improve cross-border links (road, rail, waterways) within the European Union [27]. Thus, it is possible to subdivide ports into core ports and 
comprehensive ports with regard to the identified key ports in the sense of the TEN-T. According to Philipp et al. [10], core ports represent large ports, while the comprehensive ports are medium-sized ports. Vice versa, small ports do not belong to the TEN-T. In order to reach this ambitious objective - developing an integrative Trans-European Transport Network (TEN-T) - the European Commission has launched the financial instrument "Connecting Europe Facility" $(\mathrm{CEF})^{\dagger}$. The CEF provides around $€ 24.2$ billion for the current planning period 2014 to 2020 . This budget is provided for the expansion of transport infrastructure, and thus, among other things contributes to meet the infrastructure requirements in conjunction with the development of the hinterland of the selected seaports [28].

\subsection{Liquefied Biogas as an Emission Compliance Biofuel for Shipping}

Just a few studies record approaches on the usage of LBG in the maritime sector. Similar as liquefied natural gas (LNG), the main energy carrier in LBG is methane [29]. However, the reason why LBG is a biofuel or renewable energy source is the fact that the methane is produced from biomass, that is biogas, either by anaerobic digestion or by gasification followed by methanation [30], [31]. Hence, when biogas is upgraded in order to produce fuel, it is often labelled as bio-methane [32]. Biogas or bio-methane can be liquefied to LBG or liquefied bio-methane (LBM) in the same way as natural gas to LNG [30]. LNG and LBG are nearly identical. Thus, using LBG instead of LNG in ships does not pose any additional technical problems [33]. In this context, it needs to mentioned that there exist also blended fuels on the market (e.g. mixture of LBG and LNG), which are often labelled as biofuels, too. However, in the frame of the present study, whenever LBG is mentioned, it is considered that it is produced to $100 \%$ from biomass and thus, represents a "real" biofuel (i.e. not blended with fossil natural gas - LNG). Following the study from Bengtsson et al. [30], LBG as a fuel for ships has the best overall environmental impact - concerning acidification, eutrophication, human health damage of $\mathrm{PM}_{10} /$ human toxicity and photochemical ozone formation potential, compared to LNG, HFO, MGO, rapeseed methyl ester (RME), etc. This is mainly caused by lower emissions of $\mathrm{NO}_{\mathrm{x}}$ during combustion in engines. Moreover, they concluded that LBG is better than LNG due to its lower life cycle emissions of greenhouse gases (GHG). Comparing it with marine diesel oil (MDO), the switch to LBG would cut GHG emissions by 75-98 \% - with assumed zero $\mathrm{CH}_{4}$ slip [33]. In addition, MDO's total acidifying emissions are almost double compared to LBG. Furthermore, particulate matter (PM) emission from LBG is $80 \%$ lower than in case of MDO. This is deeply rooted in the fact that LBG has a low sulphur content and simple fuel molecule, which burns with low soot and PM formation [33], [34]. Moreover, as stated by Spoof-Tuomi and Niemi [33], the combustion of liquefied bio-methane (i.e. LBG) exhibits a neutral recirculation loop for $\mathrm{CO}_{2}$. Hence, LBG has the potential to reduce significantly the impact of shipping emissions on local air quality. To sum up, LBG is an attractive low carbon alternative [33], that matches the strict SECA regulations as well as up-coming Nitrous Oxide Emission Control Area (NECA) requirements in 2021 [7], [35]-[37].

\footnotetext{
${ }^{\dagger}$ The CEF clearly describes which measures and projects are promoted and to which extent. Studies on all transport modes are subsidized with $50 \%$, whereby traffic management systems, new technologies and innovations are supported with $20 \%$ of the investment costs. In the case of construction projects, the funding rates are not defined explicitly and thus, can differ. The highest funding grants are possible in the case of waterway and rail routes with up to $40 \%$ [28].
} 


\subsection{Maritime Energy Contracting and Field-To-Ferry Concept}

Energy contracting models are well-known from the real estate or housing sector [38]. Especially in system-relevant buildings (e.g. hospitals), they play an important role, since secured energy supply at all times is crucial [39]. Next to the Energy Performance Contracting (EPC) model, especially the Energy Supply Contracting (ESC) approach is used in practice. The ESC model provides the necessary basic energy and is used for services that lack capital investments [24], [39]. The idea to adopt the ESC model in the maritime sector is quite new and had been firstly proposed by Olaniyi et al. [13], [24], [25]. Nevertheless, in their studies the crux of the matter were the fuel producers, who should apply the developed MEC model for either an efficient supply of LNG to ship-owners or a wide usage and installation of scrubbers on board of ship-owners' vessels. Thereby, the used comparison fuel was MGO, which does not comply any more with the corresponding sulphur emission regulations. However, especially by considering the LNG case, next to an exclusive focus on the downstream value chain, they do not take much regard to the ports, where the fuel supply to the vessels takes usually place. In this context, it must be clarified that not the fuel producers are empowered to decide whether a new bunkering possibility will be implemented in a harbour - such decisions are made by the port authorities and/or operators or corresponding municipalities in smaller ports. Thus, next to policy makers, the key players for an efficient transition towards supply and utilisation of alternative fuels like LNG, LBG, etc. are the seaports. Moreover, for the production and supply of biofuels like LBG, traditional fuel producers that typically focus on fossil fuels are not (necessarily) needed; as shown by the field-to-ferry business concept, which was elaborated in the frame of the GoLNG project and theoretically proposed for the Danish island and municipality Samsø. However, this study lacks of economic reasoning and thus, highlights only non-monetary environmental and social benefits of using LBG as a fuel for shipping [40]. Among other things, this can be traced back to the problem to supply LBG at a competitive supply price in comparison to LNG. Hence, it must be clarified that without any economic advantages that result in reliable incentives, the transition from fossil fuels and resources to ecological sustainable emission compliance biofuels and renewable energy will not be safeguarded. Accordingly, if subsidies are not granted for biofuels like LBG, a transition towards fossil free shipping (i.e. green shipping) is less likely.

\subsection{Blockchain and Smart Contracts}

Blockchains are distributed ledger systems that ensure via alphanumerical code and private key safe execution of transactions in a network with potentially untrusted participants. The distributed virtual ledger system can supplant physical documents as well as signatures [41]. However, one of the most important characteristics of blockchain is that for the cryptocurrency exchange intermediaries become superfluous, which automatically leads to the emergence of a decentralised trusted network [42]-[46]. Hence, through blockchain application the up-to-date information accessibility can be ensured at all times, whereat transaction and intermediary costs decrease, which is especially value adding for SMEs and entrepreneurs [47]. Thus, as highlighted by Philipp et al. [48], the blockchain technology facilitate entrepreneurial collaborations in trans-national supply chains. An advancement are smart contracts that build up on the blockchain technology and represent transactional scripts or protocols that include terms and conditions of contracts [43], [46], [47], [49]. Embedded through digital codes with software on the blockchain platform, they automatically process the negotiated contract conditions to which all involved parties previously agreed [44]. In 
addition, they are empowered to perform reconciliations that are self-executable induced through new transactions as well as updated or uploaded data and documents on the blockchain ledger - e.g. via IoT applications [41]. Hence, if all pre-defined terms and conditions are fulfilled, smart contracts automatically trigger a certain number of transactions or actions [50]. Therefore, smart contracts are able to read from and write on the blockchain [41], [48], [51]. Since they are implemented on the blockchain platform, smart contracts face the same high data security characteristics - e.g. protection against distortions, revisions and manipulations as well as deletion [44]. Nevertheless, the main advantage can be seen in the elimination or reduction of intermediary levels (e.g. third parties, middlemen, brokers or agents like governments, banks, lawyers, etc.) in diverse business activities, which fosters the efficiency and redesign of complex business structures that are characteristic for supply and value chains [41], [48]. To sum up, the unique characteristics of the blockchain technology and especially the possibility for the execution of smart contracts have promoted the ideas for even greater application areas in different markets that go far beyond the financial sector.

\section{Methods ANd Methodology}

In the framework of the given study, the theory-based and practice-related research has been applied building upon empirical data from expert interviews, case studies, observations and practical findings that have been collected and produced in the frame of the GoLNG and the ongoing Connect2SmallPorts project. The Connect2SmallPorts project focusses on improving cross-border connectivity for a functional blue and green transport area, with the objective to enhance the quality and environmental sustainability of transport services in South Baltic Sea Region, with a specific emphasis on digitalisation issues in small and medium-sized seaports - particularly blockchain and IoT. The project is part-financed by the ERDF as it is implemented in the framework of the INTERREG V A South Baltic programme. In contrast, the GoLNG project focalized on the implementation of the EU Clean Fuel Strategy and the EU Directive on Deployment of Alternative Fuel Infrastructure through technology and knowledge transfer in LNG related business activities and improvement of the LNG value chain in the BSR, whereby also the possibility of LBG production and distribution provided in the integrated LNG value chain was analysed (LBG sustainability for LNG infrastructure). The project was also part-financed by the ERDF, since it was implemented in the framework of the INTERREG V B Baltic Sea Region programme. Through active collaboration in the GoLNG project and leadership in the Connect2SmallPorts project, the researcher received access to a wide range of data and insights on the research topic, which was crucial and the stepping-stone for the current research study.

Data collection activities comprised initially the search, identification and analysis of secondary data that was gathered through the study of fuel market reports, topic-related policy regulations and guidelines, scientific literature and websites regarding up-to-date and historical spot prices. In addition, structured expert interviews with top-level managers from Karlskrona seaport were conducted in January 2020. The interviews lasted about one hour and were recorded and transcribed. The interview analysis was conducted according to Kvale [52] and Miles et al. [53].

The field-to-ferry-concept from the GoLNG project and the MEC concept from Olaniyi et al. [13], [24], [25] are used as a baseline for the development of the new LBG MEC business model. For the creation of the business model, the Business Model Canvas from Osterwalder and Pigneur [26] was applied. For the design, discussion and presentation of an innovative business model, Osterwalder and Pigneur [26] in their Business Model Canvas 
refer to nine basic building blocks: customer segments, value propositions, channels, customer relationships, revenue streams, key resources, key activities, key partnerships, cost structure. All these nine building blocks need to be described and intertwined with each other in order invent a new competitive business model. Thereby, for presenting the monetary aspects, a comparative analysis with ULSFO was carried out. For this, a case study approach according to Yin [54] was used to exemplify the operating principles of the business model. The case study refers to the comprehensive seaport Karlskrona in Sweden and the RoPax ferries from Stena Line that travel back and forth to Gdynia seaport in Poland (i.e. SECA in the BSR).

\section{Results}

Competition in the shipping sector is steady increasing due to decreasing freight rates and volumes of passengers. Hence, ship-owners - especially those, who are mainly active in emission control areas through Short-Sea-Shipping (SSS) - are under financial pressure and thus, fear retrofits of vessels' engines for complying with emission regulations, since they are associated with high investment costs and risks. To tackle this issue, in the course of the new business model, the port will henceforward provide LBG to ship-owner's vessel (i.e. energy/fuel supplier role) and will become responsible for arranging the retrofit of the vessel towards a dual fuel engine that is also able to combust LBG, as well as the subsequent maintenance (i.e. energy/fuel service role). As a result, the emission compliance costs for the ship-owner decrease and the business scope of the partner port will be enlarged through new additional service offers. Next to the resulting costs for a steady LBG supply, engine retrofit and regular maintenance, the envisaged contract (i.e. LBG MEC) will also consider an adjustment premium for the port as energy and service provider.

\subsection{Value propositions}

The superordinate value proposition is the significant contribution to the reduction of emissions as well as the sustainable switch from fossil to biofuel or renewable energy, respectively. On the one hand, in the context of the ship-owner, this means, receiving access to emission compliance fuel, at reduced or eliminated risks, investment (retrofit) and operational costs (fuel and maintenance costs). This is, because the port will become responsible for arranging the retrofit towards a dual fuel engine that is also able to combust LBG. Hence, capital investment and associated risks are taken over by the port. Furthermore, under the premise that LBG as a biofuel for ships can be supplied at approximately the same price as LNG, it is much cheaper as other usual SECA compliance fuels like LSMGO and ULSFO. This aspect can be highly beneficial, since 50 to $60 \%$ of voyage operational cost are attributable to fuel costs [55]. Accordingly, the port guarantees the steady fuel supply during the contract period. Moreover, operational costs decrease even more, since responsibility for arranging regular engine maintenance will be taken over by the port as well. Thus, port's value proposition to the ship-owner can be regarded as an "all-round carefree package".

On the other hand, the port, further potential customers and other stakeholders in the local or regional costal area benefit from fewer emissions, which contributes - for instance - to a lower occurrence probability of respiratory and cardiovascular diseases, and thus, adds substantial value to the society - but also to the environment in general. 


\subsection{Customer Segments}

The main customers are ship-owners, shipping companies or ship-operators, who have to show compliance to IMO emission regulations and fear retrofits due to high capital investments as well as related risks or uncertainties, respectively.

There exists also the potential to extend the LBG MEC business model to other transport modalities in the near proximity of the port. For instance, LBG or compressed biogas (CBG) can be used as well in the road transport sector (e.g. trucks and busses). Furthermore, it is also possible to expand the scope of customers to the industrial sector (i.e. companies with high-energy demand) and local or regional gas grid operators in the catchment area of the port in order to serve the energy demand - e.g. in the municipality. For several years, there is an increasing environmental awareness in society, so that private households are willing to pay for green gas.

\subsection{Customer Relationships}

The port and the ship-owner will become close business partners with a strong relationship that will be contractually fixed. The "all-round carefree package" includes and enables (1) a guarantee for steady LBG supply, (2) takeover of responsibility for arranging dual fuel engine installation and regular engine maintenance, (3) risk absorption, (4) added value through emission compliance solution, (5) renewable fuel branding (i.e. marketing), as well as (6) expertise and consultation regarding planning and execution of the MEC project. The shipowner is the main costumer in the LBG MEC business model. Thus, the port has a special interest to optimally satisfy the needs of the ship-owner. Moreover, since the retrofit is the investment of the port, during the contract period, the legal ownership regarding the dual fuel engine belongs to the port, whereat the ship-owner receives the economic ownership. Nevertheless, as soon as the contract period ends, the legal ownership goes over to the shipowner, too, whereby in case of an open residual value, the ship-owner has to arrange a corresponding one-time compensation payment - usually whenever the contractual relationship ends prematurely. Hence, regular exchange of information as well as monitoring activities must be ensured. This includes regular inspections and maintenance measures in order to secure long-term and perfect engines operations that are initiated and arranged by the port in order to optimally fulfil the needs of the ship-owner as a primary customer (i.e. securing ship-owners' primary business operation - shipping). Thus, the port has the responsibility to keep under constant review all engines - i.e. control function. All these aspects must be negotiated, defined and agreed between both contractual parties prior the contract is closed (i.e. signing process conducted by the contractual parties).

In the potential event that the LBG and biogas clientele will be enlarged over the time to other transport modalities, the industrial sector or gas grid operators, this will be achieved always on a contractual basis, whereby the interdependency and scope of required services define the business relationships.

\subsection{Key Partnerships}

Next to the ship-owner, who will be the main costumer, there exist different key partnerships that must be established by the port. In order to identify all relevant key partners, two potential cases must be considered: (1) the port focus on the organisation of the downstream LBG value chain, and (2) the port considers to integrate horizontally in the upstream LBG value chain, too. 
Initially, in order to kick-start the new LBG MEC business model, the port will focus on the downstream LBG value chain - i.e. primarily on the ship-owners as the main end-users. Accordingly, the port primarily concentrates on the steady supply of LBG to ship-owners' vessels. In this case, the supply of LBG to the port requires a key partnership with a LBG bunker trader (i.e. intermediary - fuel broker) or directly with a biogas liquefaction company, who will be responsible for the overall LBG supply to the port. Since the port will be also responsible for arranging the retrofits towards dual fuel engines as well as the related regular maintenance, an additional key partnership is needed with a shipyard or maintenance company, respectively. Vice versa, all this induces the necessity of additional partnerships with an investment bank and an insurance company.

However, in case of a developed and enlarged group of customers in a maturity stage, a stronger focus on the upstream LBG value chain can make sense. In this case, additional longterm partnerships (e.g. with additional customers - road transport sector, industrial companies, etc.) must be established by the port, whereby other partnerships become superfluous (i.e. reduction or elimination of intermediary levels by the port - replacement). The general upstream LBG value chain as it is shown in the Samsø field-to-ferry case consist of: farming system, biogas plant, biogas upgrading and liquefaction. The major advantages of an integration of the port into the upstream LBG value chain can be seen in higher control, short transportations, more reliable value chain, exclusion of intermediaries, and thus, decreasing transaction and enforcement costs, whereby LBG or biogas can be supplied at lower prices to the end-users. Hence, new key partnerships emerge with the local or regional farmers, whereas also expertise and support through key partnerships with suppliers of biogas plants as well as upgrading and liquefaction technologies is needed (i.e. for the construction project and subsequent plant maintenance activities). Vice versa, previously important key partnerships like LBG bunker trader or liquefaction company become superfluous - i.e. elimination of intermediary levels. On the other hand, this scenario results in high investment costs for the port. Nevertheless, according to the findings from the GoLNG project, the overall investment for the development of the needed infrastructure - comprising biogas plant as well as biogas upgrading and liquefaction facilities - amounts about $€ 10$ million [40]. On a first glance, this amount appears to be high, but considering that this covers the establishment of an entire local or regional LBG value chain, it can be evaluated as low. In addition, national or international subsidies can be used to co-finance this construction project. For instance, according to the CEF (TEN-T), funding rates between 20 to $50 \%$ are achievable [28]. That this is feasible, can been seen in the Frederikshavn case, where Nordliq Nature Energy, Bunker Holding, Kosan Crisplant and Frederikshavn Havn are partners in the consortium behind Nordliq, a liquefaction plant project in the port of Frederikshavn (Denmark) with a targeted LNG production capacity of 50,100 or 150 metric tonnes per day [40]. ${ }^{\star}$ This project receives support from the European Commission via the CEF. In addition, for the biogas production, the bio waste from the region can be used as well, which opens opportunities for sustainable and better waste handling. In addition, in case of an existing local or regional gas grid, which was established for suppling natural gas to the surrounding household area and industrial companies, the same infrastructure can be used for the provision of biogas. Hence, a key partnership with the local or regional gas grid operator or owner can be reasonable, whereby also the port can cover its own energy demand. Moreover, as a side effect, the farmers can receive an organic fertilizer after the biogas process. Hence, keeping these aspects

\footnotetext{
$\$$ According to the project in the port of Frederikshavn, LNG will be supplied from the process tanks by tank trucks to the loading points, whereby LNG will be offered to ships and heavy load trucks [40].
} 
in mind, the LBG MEC business model in an enlarged case has the potential to contribute substantial to the creation of a circular economy. Lastly, the business model can contribute as well to the development of a small energy autarkic region as well as the emergence of a green transport corridor.

\subsection{Key Activities}

In the initial case, the key activities of the port are enlarged through the new biofuel provision (here: LBG) and additional services that refer primarily to the arrangement of the engine retrofit and subsequent maintenance. All these activities must be planned and arranged individually for each vessel - according to the respective ship features. Hence, the port needs to increase staffs' knowledge, skills and capabilities regarding LBG handling and project management. This can be achieved by trainings and seminars or recruitment of new employees with expertise in LBG, whereby also outsourcing is an option. In addition, in order to secure long-term and perfect engines operations and thus, to fulfil optimally the needs of the ship-owner, a regular exchange of information as well as monitoring activities must be performed. Therefore, another key activity can be seen in ensuring a secured data information exchange, by the integration and usage of a suitable technology that is also able to automatically generate contracts and billing with all relevant key partners and customers. Similar as in case of Philipp et al. [48] - who described the integration of a blockchain smart contracting system in the charter-party contracting process - also in the frame of maritime energy contracts, the usage of the blockchain and smart contract technology is reasonable since the standard MEC is also a contract that can be automated. Moreover, such a solution becomes quite effective through the inclusion by IoT sensors at the ship engines and tanks that permanently monitor the optimal performance and fuel consumption.

In the maturity stage, further key partnerships occur and thus, the scope of the abovementioned key activities will enlarge (e.g. secured data information exchange including automated contract generation to a wider group of relevant key partners), whereat new and additional key activities emerge, too. For instance, one of the new introductory key activities embraces the identification of suitable funding schemes on national and/or international level for the construction project of the local or regional LBG value chain. However, in order to initiate the project, which embraces grossly the phases planning, development and finalisation/launch of operations, the most important preparatory key activity is the generation of public awareness, which implies a comprehensive integrative communication process in the region with all relevant stakeholders. An extensive communication and a public discussion about the project in a reasonable manner are important, since such measures increase the chance of the project to be successful. Next to acceptance by the public, political support is needed for receiving legal permissions.

\subsection{Key Resources}

By focusing on the downstream LBG value chain, a new key resource is the LBG, which will be provided to ship-owners' vessels. This includes as well the needed fuelling systems for pumping LBG into vessels' tank. Next to this, since the port takes over the investment for engine retrofit, the resulting dual fuel engine plus LBG ship tank including pumping system, etc. will become new key resources, too. Compared to these tangible key resources, the intangible key resources embrace the LBG experts and service personnel in the port, if outsourcing is not considered. Moreover, the blockchain with smart contract applications for 
secured data information exchange, as well as the IoT sensors at the new dual fuel engine and tank for monitoring purposes represent further intangible and tangible key resources.

If the port considers to integrate into the upstream LBG value chain, the physical infrastructure for the production and bunkering of the biogas and LBG - thus, almost the entire LBG value chain - becomes a new key resource (i.e. especially the biogas plant, biogas upgrading and liquefaction facilities as well as the established bunkering option in the port). In addition, the associated competence in form of staff along the local or region LBG value chain becomes an intellectual key resource, too. Since, this scenario opens also the possibility to sell biogas or CBG to further end-users, these outputs as well as related supply facilities or equipment, respectively, represent further potential key resources.

\subsection{Channels}

Regarding the downstream LBG value chain, the port primarily concentrates on the steady supply of LBG to ship-owners' vessels. Similar as in the case of LNG, for bunkering and supply of LBG in a port, there exist four general opportunities that cause different investment costs [56]: ship to ship transfer (STS), truck to ship transfer (TTS), terminal/pipeline to ship transfer (PTS), and portable tanks. In the latter case, as soon as the tank is empty, it will be replaced by a full tank; hence, the reception of LBG to the port consists of loading and unloading as well as connection and disconnection of the portable tank system [57]. Especially the TTS and portable tanks options tie up little capital and are suitable for the supply of small quantities of LBG. However, in the initial case, the overall supply of LBG to the port is outsourced. Hence, the port staff is responsible for fuelling the LBG from the bunker option into vessels' tank. LBG distribution and inventory can be monitored automatically through the implementation of blockchain as well as smart contract applications, which additionally bears the potential to eases the management of the value chain. Hence, for enhancing the LBG MEC business model, a blockchain driven smart contracting system is proposed. Through this, the material, information and financial flow of the overall LBG value chain can be improved. The maritime energy contracting process embraces three phases: (1) pre-fixture, (2) fixture and (3) post-fixture.

In the pre-fixture phase, relevant information that is needed from the ship-owner are ship type, size, current engine status, annual runtime and further features of the vessel. A smart contract application can conduct initial calculations for the whole project according to the provided data by the ship-owner about the vessel. Hence, the smart contract application can automatically compute and estimate the needed capital investment for the retrofit towards a dual fuel engine based on the ship characteristics, as well as deliver an answer whether the investment is reasonable due to ships' age, size, available space for dual engine and tank installation, the intended purpose, etc. Additional input data that is needed for the smart contract investment calculation is stored on the blockchain, e.g. price range for retrofits towards dual fuel engine according to different ship types - provided by the shipyard (key partnership). Furthermore, the smart contract application can also predict the needed average regular LBG quantity, maintenance and inspection schedule including potential average costs that occur, as well as the LBG supply price - derived from current data, which can be integrated, stored and automatically self-executable updated on the blockchain ledger in realtime. Building upon this, a kind of pre-contract can be automatically generated by the smart contract application, since the provided data from the ship-owner represents the initial information and pre-conditions that must be reconciled and fulfilled for the aspired development of the LBG MEC. 
In the fixture phase, the MEC will be signed by both parties (i.e. port and ship-owner) insofar all contract terms and conditions are negotiated and subsequently agreed. In an optimal case, the MEC contains all relevant information, e.g. details regarding the ship-owner and port, ship details, LBG price (range), expected average fuel demand, inspection and maintenance schedule, quality assurance, interest rate, adjustment premium for the port, contract duration, in case of a fixed route (origin and) destination port(s), payment method, payment terms and details including currency, clauses in case of a potential prematurely contractual ending as well as the corresponding calculation method and procedure of the resulting necessary one-time compensation payment, and other obligations and rules as well as clauses. Therefore, also legal aspects and responsibility in case of unexpected events or in case of non-performance have to be integrated into the MEC. Once the port and the shipowner have jointly agreed on the rates and terms, the MEC agreement will be fixed through the signing process. This procedure can be automated by a blockchain smart contracting system, too. Norta [58] developed a smart contracting setup lifecycle for negotiation procedures, which can be adapted through reconfiguration and thus, applied in the course of the MEC negotiation process. Furthermore, current data like biogas price, which usually function as input data and a point of orientation, can be automatically integrated in the smart contracting negotiation process. Hence, if all pre-defined conditions are fulfilled (i.e. the smart contracting setup lifecycle for negotiation achieves a concluding outcome), the smart contact application is able to automatically generate the smart MEC, whereby the contract parties can digitally sign via a private key. Thus, the smart contract application replaces the physical paperwork (i.e. multiple versions of files, signatures, etc.), whereat each party can digitally validate files and generate copies. Moreover, the elaborated and fixed MEC as a smart contract is than automatically stored and secured on the blockchain. Accordingly, the smart MEC becomes decentralised and distributed secured, fraud-resistant, irreversible, steady verifiable, retraceable and retrievable at all times for all involved parties during the contract period.

Next to the one-time engine retrofit, the post-fixture phase mainly embraces monitoring tasks. These monitoring tasks primarily refer to the fuelling of the ships (i.e. distribution of LBG) and maintenance. Normally, both processes cause much physical paperwork. For instance, the status information about the regular fuelling operations result usually in the back and forth sending of physical documents and copies between the port and ship-owner (i.e. port staff that is responsible for the fuelling, the ports' controlling department, port/ship agent(s), ship's captain and crew staff, etc.) that subsequently function as important input data for the regular fuel supply billing. However, the implementation of a blockchain smart contracting system with IoT applications can also improve the fuelling as well as maintenance scheduling, since all corresponding information and assisting documents can be uploaded and stored on the blockchain in real-time; and thus become decentralised secured, fraud-resistant, immutable, transparent, permanently auditable ${ }^{\S}$ and historically retraceable as well as accessible for smart contract applications as well as all involved participants, who have

\footnotetext{
$\S$ Smart contracts can automatically perform in real-time auditing procedures in case of uploaded documents and other types of information on the blockchain. This includes as well the automated checking and programmed processing of transactions or other kind of information that occur and at the same time can lead to the fulfilment of specific pre-defined conditions, which induces triggering of further actions or transactions (i.e. if-then-else functions). On the other hand, corresponding users are able to upload data and approve the uploaded documents and information from other entities - if permission or rights are given. Hence, next to data reading permissions, some or all blockchain participants can have a writing permission, too. Security is guaranteed through the hashing as well as validation and verification mechanisms in the frame of regular block creations for the chain. Thus, all or only selected parties receive a copy of the blockchain ledger for auditing purposes. Therefore, data fraud or tampering becomes impossible.
} 
permission. In general, this fosters trust among all involved actors and enhances efficiency of the processes due to a higher flexibility.

Regarding maintenance, recent technological innovations allow for optimised scheduling of activities. For instance, acoustic sensor applications are nowadays capable to show up disturbances in engines operations that are no longer perceptible to human hearing. Hence, engines and pumping systems can be permanently monitored by IoT applications in a much wider frame than current electronics on a usual ship can do. Furthermore, monitoring operations during the voyage over long distances (e.g. from the port terminal) become possible. This ensures that maintenance is induced before a serious problem arises that can lead to reduced performance or in a worst-case scenario to a breakdown. Such applications are summarised under the term "smart maintenance" and are highly value adding due to cost savings - especially in different industrial sectors that are dependent from smooth functioning of machines and facilities at all times. Smart maintenance can cause spontaneous or shortterm, but rather small interventions that in total are much cheaper than the overall costs that may arise from a complete production breakdown or performance dropout, which among other things result in shortages and regular more expensive maintenance or purchases of new engines, machines or even complete facilities. Hence, smart maintenance is value adding to customers and guarantee sustainability in resource management, which vice versa protects the environment. To sum up, IoT and smart contract applications feed the blockchain with needed input data and smart contracts process these information and data in order to trigger further transactions or actions - e.g. initiations of spontaneous inspection or short-term maintenance, respectively.

Regarding the fuelling of the ship, the IoT sensor applications on board at the tank can monitor and send the fuel consumption and need in real-time to the port. Furthermore, the delivery of LBG from port bunker to ships' tanks can be also monitored by the blockchain smart contracting system (i.e. controlled distribution). Hence, when the ship is in the port and needs fuelling, the back and forth sending of information, documents and copies for the overall process is not needed any more. Thus, the integration of blockchain smart contracting system bears the potential to foster the establishment of full- or semi-automated LBG bunkering and fuelling operations in the port [56]. Moreover, blockchain and smart contracts enable automatically forecast calculations and the triggering of LBG repeat orders, which secures the avoidance of shortages in supply in the port.

Bearing this latter aspect in mind and transferring it into the context of a potential enlarged case, where either all actors of the value chain participate in the same blockchain (i.e. joint digital infrastructure ${ }^{* *}$ ), or the port considers to develop a local or regional LBG value chain as an integrator, the blockchain smart contracting system is able to optimise the management of the entire chain. With other words, through blockchain and smart contract applications, the upstream LBG value chain nodes and entities can receive real-time information and predictive data about the current as well as expected future consumption or demand of the downstream LBG value chain - including end users. Accordingly, with blockchain smart contracting systems value chain management becomes smarter and thus, more efficient, since resourceoptimised production along the whole chain is ensured through automatically generated realtime and forecast data. Thus, over and under capacities can be avoided.

\footnotetext{
** By using blockchain as a joint infrastructure data exchange between incompatible systems is avoided and the data transfer becomes digital and automated, which enables a simultaneous document processing and process tracking and thus, a greater transparency and flexibility as well as streamlined processes, since no back and forth sending of physical documents is necessary.
} 
Moreover, this includes as well the possibility that smart contracts are able to automatically conduct the financial calculations and the related generation of billings and payments, e.g. for the famers, who produced the biomass for the biogas plant, as well as for fuel and energy supply of downstream stages of the value chain or end-users. This is achieved through the underlying blockchain, where the needed input data is available through IoT applications and integrated market data as well as the smart contracts - that include all respective rates, prices, terms, etc. - along the chain. The same applies for the planned or spontaneously induced inspections and maintenance of tangible key resources; or if a contractual relationship with an end-user (e.g. ship-owner) prematurely ends, since the corresponding smart contract application is able to perform automatically the corresponding calculations (e.g. residual value and thus, necessary one-time compensation) and subsequent generation of the billing. Hence, all occurring financial calculations, billings and transactions along the LBG value chain can be automatically elaborated and triggered, if all corresponding pre-defined conditions are fulfilled and thus, all relevant data and information is available in the chain of blocks through uploaded, secured and shared files. As a result, the emergence of objections by the involved parties can be limited due to the decentralised nature of the blockchain and smart contract technology. Accordingly, this procedure guarantees a trustful and fair approach for automated concluding calculations as well as monetary transactions, which decreases costs due to shortened process time and lower manual works. Therefore, it can be stated that blockchain and smart contracts are able to contribute substantial in the pursuit of perfection of material, information and financial flows in supply and value chains.

Nevertheless, apart from all these showcased benefits, in particular the potential exclusion of intermediaries can have far-reaching positive impacts. The formerly given trust through the presence of the intermediaries is compensated by the decentralised nature and further benefits of the blockchain and smart contract technology, which automatically leads to the emergence of trust among all involved users of the digital infrastructure (i.e. blockchain). Through the exclusion of the intermediaries, transactions and enforcement costs decrease which is value adding for the port as an integrator, but also indirectly for all participants in the blockchain network through potential spill-over effects. Especially for smaller and entrepreneurial actors this enables participation [48].

Lastly, for the described purposes, a private permissioned blockchain would be an appropriate solution. This is reasoned by the fact that within a permissioned blockchain, it is possible to clarify access and modification rights of the participants as well as proof of personal identification. Next to this, in a private blockchain, specific transactions or actions can be set as private to certain participants. This can be regarded as a data filter, which ensures that only certain authorised participants are allowed to add, receive or inspect corresponding data and information that is needed for further actions in their area of responsibility [48].

\subsection{Revenue Streams and Cost Structure}

To exemplify the operating principles of the proposed LBG MEC business model, in the following, the initial case for kick-starting the business model - where the port and shipowner become close business partners - will be analysed in detail by examining the related revenues and costs. As proposed by Olaniyi and Gerlitz [25] as well as reasoned by the fact that a vessel represents a moveable asset, the nominal contract duration should be about five years, whereby regular audits shall be performed, which may result in necessary contract adjustments. The LBG MEC embraces three parts: (1) LBG baseline price, (2) adjustment, and (3) asset costs (i.e. costs for retrofit towards duel fuel engine and subsequent maintenance). 
In order to highlight the different components of the LBG MEC, the case study of Karlskrona seaport in Sweden is used. Karlskrona is a comprehensive port according to the analogy of the TEN-T and thus, the port may receive financial support through the CEF for infrastructure developments in the frame of a potential expansion scenario. On the other hand, this means that the port of Karlskrona can be classified as a medium-sized or regional seaport. In 2019, the seaport handled ca. 450000 tonnes of freight and 700000 passengers. Therefore, it can be noted that Karlskrona represents rather a typical ferry port. The single regular ferry route is to Gdynia seaport in Poland. Hence, the ferry operation refers exclusively to the BSR and thus, SECA. The route is operated by Stena Line with the three RoPax ferries "Stena Vision", "Stena Spirit" and "Stena Baltica". The characteristics of the three ships are quite similar, and currently all of them use ULSFO. Each of the three vessels sails about 300 to 350 times per year, whereby one voyage (back and forth) takes ca. $2 \cdot 10$ hours and causes a total fuel consumption of about 53.4 to 62.3 metric tonnes (MT). Stena Line has a high interest in alternative fuels, whereby the company considers especially LBG as the shipping fuel of the future [59]. For the following calculations, it is assumed that LBG is used to $100 \%$ after retrofit, whereby initial calculations refer to one ship.

Regarding LBG baseline price, currently there exist no market data (neither historical nor up-to-date), since the LBG market is not sufficiently developed. Nevertheless, if LBG should become a competitive alternative to LNG, the LBG baseline price has to be approximately the same as for LNG, which can be achieved in the long-term most likely only through subsidies. However, in order to derive an approximate, but reasonable LBG baseline price, the study from IEA [60] is used. According to this study, landfill gas recovery systems (2000 $\mathrm{m}^{3} / \mathrm{h}$ ) can produce biogas for less than $3.00 \$ / \mathrm{MBtu}$, whereby upgrading costs amount 2.00 to $4.00 \$ / \mathrm{MBtu}$ for facilities that upgrade around 3.5 million $\mathrm{m}^{3}$ of biogas per year. By taking the average upgrading costs yield total biogas supply costs of $6.00 \$ / \mathrm{MBtu}$, which is equal to $211.86 \$ / \mathrm{m}^{3}\left(1 \mathrm{MBtu}=0.02832 \mathrm{~m}^{3}\right)$. However, since liquefaction costs are also unknown, the corresponding finishing factor is deduced from the American export price spread between natural gas and LNG from EIA (Independent Statistics and Analysis - U.S. Energy Information Administration) [61], since biogas can be liquefied to LBG in the same way as natural gas to LNG, which pleads for a similar finishing factor. The resulting average finishing factor for January 2018 is $1.12727 .{ }^{\dagger}$ By taking the corresponding dollar exchange rate $(€-\$)$ at the end of the same month with 1.2413 [62] and the liquid density factor of methane with $0.42 \mathrm{~kg} / 1$ yields an LBG baseline price of $458.10 € / \mathrm{MT}$ at the end of January 2018 t!

To determine the asset costs, as a starting point, the corresponding data for a general LNG retrofit is used, since LNG and LBG are nearly identical concerning engine's fuelling and combustion, and thus, using LBG instead of LNG in ships does not pose any additional technical problems, which at large pleads for similar costs. According to Balland [63], the

\footnotetext{
${ }^{\dagger}$ The average price of U.S. natural gas pipeline exports in January 2018 was $3.85 \$ / \mathrm{Tft}^{3}$, whereby the average price of liquefied U.S. natural gas exports in the same month was $4.34 \$ / \mathrm{Tft}^{3}$ [61]. The resulting finishing factor is relatively opportune, since the price spread was usually higher during the last decade. However, since the U.S. export LNG price can be also lower than the related natural gas price (e.g. usually between January 2003 to August 2008) [61], the average price spread as of January 2018 was used for the approximation.

$\$$ Usually the LBG baseline price can be higher; but in some cases also lower, due to volatility in dollar exchange rate and finishing factor (i.e. average price spread factor between LNG and natural gas). Moreover, according to the study of IEA [60], total baseline supply costs for biogas vary according to the used feedstock and technology. Hence, the LBG supply price can be usually higher, but in order to generate a reasonable approximate value for the LBG baseline price and to avoid hypothetical assumptions about possible subsidies that would result in a competitive market price in comparison to LNG, the LBG baseline price was derived as described and set with $458.10 € / \mathrm{MT}$.
} 
usual total investment costs for a retrofit towards dual fuel engine that is also able to combust LNG (or LBG, respectively) amount about \$ 5800000.00 to 6100000.00 or $€ 4672520.74$ to 4914202.85 , respectively (by using the dollar exchange rate at the end of January 2018). On the basis of the average total investment costs for the retrofit together with an economic lifetime of 15 years and an interest rate of $6 \%$, the average annual asset value amounts $€ 493$ 537.78. According to the study from Madsen [64], the annual total maintenance and repair costs of comparable ships (i.e. with a dual fuel engine that is also able to combust LNG or LBG, respectively) range between $\$ 124843$ to 301408 or $€ 100574.40$ to 242816.40 , respectively (by using the dollar exchange rate at the end of January 2018). By taking the corresponding average value of the annual total maintenance and repair costs plus the average annual asset value, the average annual project asset costs amount $€ 665233.18$. The asset costs are derived from the proportion of the average annual project asset costs at the average annual LBG consumption (i.e. in the latter case: average annual voyages multiplied with the average LBG consumption per voyage). The average annual LBG consumption is 18801.25 MT. Thus, the asset costs yield $35.38 € / \mathrm{MT}$.

For calculating the adjustment, the following formula is applied:

$$
A_{L B G}=\frac{P A C_{L B G} \cdot\left(0.5+0.3 \cdot \frac{C P I_{t=i}}{C P I_{t=0}}+0.2 \cdot \frac{L P I_{t=i}}{L P I_{t=0}}\right)}{C_{L B G}},
$$

where

$\begin{array}{ll}A_{L B G} & \text { LBG adjustment; } \\ P A C_{L B G} & \text { LBG project asset costs; } \\ C P I_{t=i} & \text { Consumer price index at } t=i ; \\ C P I_{t=0} & \text { Consumer price index at } t=0 ; \\ L P I_{t=i} & \text { Labour price index in } t=i ; \\ L P I_{t=0} & \text { Labour price index in } t=0 ; \\ C_{L P G} & \text { LBG consumption; } \\ t=i & \text { Contract point in time; } \\ t=0 & \text { Contract starting point in time. }\end{array}$

According to the formula, within the adjustment, the costs of the asset, inflation and changes in salary are considered [13]. In respect of the weighting factors, it can be stated that $50 \%$ of the adjustment is stable, $30 \%$ depends on the inflation (consumer price index), and $20 \%$ depends on the development of the salary costs (labour price index) [24].

In the frame of the example case, the adjustment is calculated for September 2018. Hence, by taking the CPI of Sweden at the end of January 2018 (322.51) and September 2018 (331.14), as well as the LPI of Sweden at the end of January 2018 (130.00) and September 2018 (131.70) [65], the adjustment amounts $35.76 € / \mathrm{MT}$.

Accordingly, the LBG MEC price (i.e. new and additional revenue per MT for the port) that would be offered by Karlskrona port to Stena Line amounts 529.24 €/MT based on the LBG baseline price, the asset costs and the adjustment. Comparing this LBG MEC price with the ULSFO price at the last trading day in September 2018 (i.e. 28 September 2018) that amounts $670.50 \$ / \mathrm{MT}$ or $577.72 € / \mathrm{MT}$ (by using the dollar exchange rate at the same trading date with 1.1606 [62]) according to the Rotterdam Bunker Prices [66], yield cost savings for Stena Line of about $48.48 € / \mathrm{MT}$. Moreover, considering the average annual LBG consumption per ship results in average annual cost savings per ship of about $€ 911484.60$ for Stena Line. Deeply 
rooted in the fact that Stena Line operates on the route to Gdynia (Poland) with three ships, the average annual cost savings for the three vessels would amount $€ 2734453.80$. Vice versa, the adjustment of $35.76 € / \mathrm{MT}$ represents the potential gross profit of Karlskrona port that would be generated by the new LBG MEC business model. Hence, by taking into account the average annual LBG consumption per ship, the average annual gross profit from the new business model for Karlskrona port would be $€ 672332.70$ per ship, whereby the average annual gross profit in case of LBG supply to all three vessels from Stena Line would yield $€ 2016$ 998.10. The results per MT are summarised in Fig. 1.

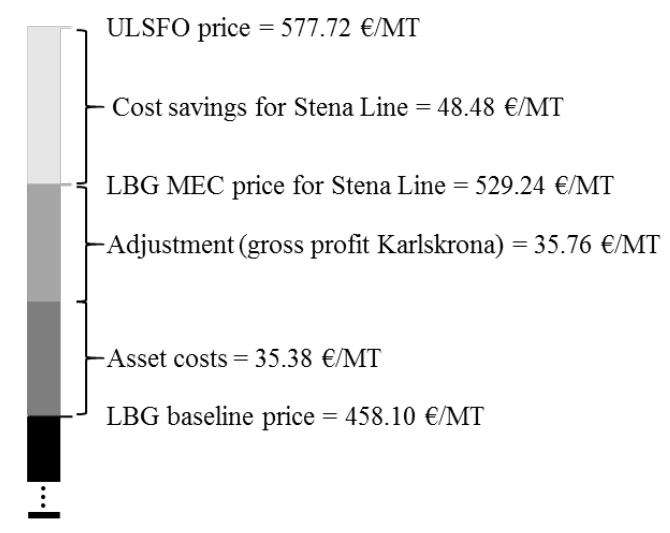

Fig. 1. Gross profits and cost savings by the LBG MEC business model.

\section{DiscuSSION AND CONCLUSION}

In this study, the possibility to supply LBG as a biofuel or renewable energy source in the maritime sector and beyond was investigated. The research revealed that the developed and proposed LBG MEC business model contributes to break through the biofuel supply and demand dilemma. However, the major challenge for LBG as a sustainable alternative biofuel for ships is the availability and supply at a competitive market price in the quantities needed for shipping [35]. As showcased in the frame of the case study, the estimated LBG baseline price can be higher, whereby due to high volatility of oil and gas prices on the market, the supply price of fossil, but emission compliance fuels like LSMFO, ULSFO, etc. can be lower, too, which may swoop the advantages of LBG utilisation. In contrast to this, a wide usage of LBG in the maritime sector is also hindered by a relatively low LNG price on the market, which can be seen as a substitute, since LNG and LBG are nearly identical, and thus, both can be replaced by each other. The use of LNG in the shipping sector is relatively widespread, wherefore the LNG bunker market is established around the globe and is expected to grow further. However, if LBG should become an alternative to LNG, LBG needs to be supplied at a competitive supply price that is approximately the same as for LNG. Otherwise, there are no economic reasons or incentives that would advocate the usage of LBG instead of LNG. Obviously, one of the most likely and realistic solutions would be subsidies that are borne by society. According to the study from Olaniyi and Gerlitz [25], the LNG baseline price was 438.60 €/MT. Hence, if LBG should compete with LNG in the long-term, subsidies must effect that the LBG baseline price level drops at least with 4 to $5 \%$. In this case, the derived and presented cost savings for the ship-owner in this study could be exceeded and form sufficient economic incentives for the desired entrepreneurial collaboration in the frame of the LBG MEC business model. 
Another point of discussion can be seen in the fact that LBG faces the same problem as LNG, since in both fuels the main energy carrier is methane. The potential methane slip, which can occur in case of an incomplete combustion in engines as well as leakages in the biogas production [30], is a problematic aspect, since methane has a global warming potential which is 28 times higher than that for $\mathrm{CO}_{2}$ over a 100-year perspective, and 84 times higher over 20 years [67]. Hence, innovative technology improvements and developments are needed for an efficient control and methane slip abatement, in order to fully exploit the potential of LBG as a renewable fuel for ships [68].

Furthermore, the research showed that blockchain and smart contracts are able to foster the implementation of the business model and optimisation of value chain operations. This is, because all actions and transactions can be optimally controlled between all involved actors through automated information exchange. The upstream LBG value chain nodes and entities can receive real-time information and predictive data about the current as well as expected future consumption or demand of the downstream LBG value chain - including end users. Hence, with a blockchain smart contracting system value chain management becomes smarter and thus, more efficient, since resource-optimised production along the whole chain is ensured through automatically generated real-time and forecast data. Thus, over and under capacities as well as bottlenecks can be avoided. Moreover, all occurring financial calculations, billings and transactions along the LBG value chain can be automatically elaborated and triggered by an implemented blockchain smart contracting system. Therefore, it can be concluded that blockchain and smart contracts are able to contribute substantial in the pursuit of perfection of material, information and financial flows in supply and value chains.

Next to the central entrepreneurial collaboration between port and ship-owner, the study investigated as well the possibility of a potential integration of the port in the upstream LBG value chain. As shown by the study, in case of a developed and enlarged group of customers in a maturity stage, a stronger focus on the upstream LBG value chain can make sense. The major advantages of a horizontal integration of the port into the upstream LBG value chain can be seen in higher control, short transportations, more reliable value chain, exclusion of intermediaries, and thus, decreasing transaction and enforcement costs, whereby LBG or biogas can be supplied at lower prices to the end-users. In contrast to this, the investment costs are acceptable for the development of a local or regional LBG value chain. Moreover, considering the potential gross profit that may arise from the LBG MEC business model, this supplementary step is reasonable and economically feasible. Especially for the case study seaport Karlskrona, from a spatial planning perspective, a bunker solution for LBG would make sense, since in the southern part of Sweden currently there exist no LNG or LBG bunker solution (cf. [69]). In Sweden, starting from Karlskrona, the nearest LNG bunker facilities are established or planned in Stockholm and Gothenburg, which both are far away. Hence, an LBG bunker option in Karlskrona could be an attractive alternative option due to the promising site factor that may generated a competitive advantage for the port. However, under the premise that LBG could be supplied at a competitive market price (i.e. in comparison to LNG) and in sufficient quantities, the LBG MEC business model can be easily adopted by other ports.

Next to the limitations that arise from the made assumptions (e.g. LBG is used to $100 \%$ after retrofit and the quantity of LBG consumption is equal to the previous consumption of ULSFO), the central limitations of the study are related to the lack of available data and lack of prior research studies on the topic (i.e. especially methodological limitations). Since so far the LBG market is not established, no exact market data could be identified. Thereby, it needs to be noted that the comparative study considered no transportation and/or storage costs for ULSFO and LBG. Irrespective of what bunker solution (STS, TTS, PTS, portable tanks) is used, generally 
bunkering of LBG and LNG is much more complex than in case of common fuels like ULSFO, since these methane fuels stay liquid only at a temperature of $-162{ }^{\circ} \mathrm{C}$, which causes proportional higher handling, transport and especially storage costs than in case of common fuels. Hence, the presented LBG baseline price was derived from secondary data and represents an approximation only. Related to this is the lack of prior research studies on the topic, since LBG as a fuel for ships has not been widely examined in the research landscape. Hence, just a few studies record approaches on the usage of LBG in the maritime sector. Accordingly, future research activities should have a closer look on LBG or other biofuels that contribute to green shipping, in order to benchmark suitable renewable fuels and to derive environmentally friendly and feasible future development perspectives for the maritime transport sector.

\section{ACKNOWLEDGEMENT}

This work has been supported by the European Regional Development Fund - ERDF (INTERREG V B: Baltic Sea Region programme \& INTERREG V A: South Baltic programme) within the projects GoLNG and Connect2SmallPorts.

\section{REFERENCES}

[1] Funke M., Yu H. The emergence and spatial distribution of Chinese seaport cities. China Economic Review 2011:22(2):196-209. https://doi.org/10.1016/j.chieco.2011.01.002

[2] Pastori E. Modal share of freight transport to and from EU ports. Policy Department B: Structural and Cohesion Policies European Parliament, Brussels: Belgium, 2015.

[3] Eurostat. Country level - gross weight of goods handled in all ports [Online]. [Accessed 31.12.2019]. Available: http://appsso.eurostat.ec.europa.eu/nui/show.do?dataset=mar_mg_aa_cwh\&lang=en

[4] Eurostat. Country level - passengers embarked and disembarked in all ports [Online]. [Accessed 31.12.2019]. Available: http://appsso.eurostat.ec.europa.eu/nui/show.do?dataset=mar_mp_aa_cph\&lang=en

[5] European Commission. Communication from The Commission Ports: an engine for growth [Online]. [Accessed 31.12.2019]. Available: http://eur-lex.europa.eu/legalcontent/EN/TXT/?qid=1467292504679\&uri=CELEX:52013DC0295

[6] International Transport Forum at the OECD. Global trade: International freight transport to quadruple by 2050. Paris, France, 2015.

[7] Gerlitz L., Philipp R., Beifert A. Smart and Sustainable Cross-Sectoral Stakeholder Integration into Macro-Regional LNG Value Chain. Proceedings of the International Conference on Reliability and Statistics in Transportation and Communication 2018:36:112-126. https://doi.org/10.1007/978-3-319-74454-4 11

[8] Philipp R., Ozarska A., Prause G. Sustainable Electronic Product Development in the Baltic Sea Region: A Regional Gap Analysis of Lab Testing Services. Environmental and Climate Technologies 2019:23(3):85-100. https://doi.org/10.2478/rtuect-2019-0081

[9] Philipp R., Prause G., Meyer C. Blue Growth Potential in South Baltic Sea Region. Transport and Telecommunication Journal 2020:21(1):69-83. https://doi.org/10.2478/ttj-2020-0006

[10] Philipp R., Gerlitz L., Prause G. Regionale Häfen auf Digitalisierungskurs: Intelligentes Wachstum und nachhaltige Wertschöpfung entlang der kleinen und mittel-großen Häfen des Ostseeraumes (Regional ports on course for digitization: intelligent growth and sustainable value creation along the small and medium-sized ports of the Baltic Sea region). Proceedings of WiWiTa 2018 Conference 2018:77-86. (in German)

[11] European Commission. Communication from The Commission to The European Parliament, The Council, The European Economic and Social Committee and The Committee of The Regions Blue Growth - Opportunities for marine and maritime sustainable growth. Brussels: European Commission, 2012.

[12] Madjidian J., et al. Developing a strategy for LNG powered transport corridors in the Baltic Sea Region. 2017.

[13] Olaniyi E. O., Gerber P., Prause G. Strategic Energy Partnership in Shipping. Proceedings of the International Conference on Reliability and Statistics in Transportation and Communication 2017:102-111. https://doi.org/10.1007/978-3-319-74454-4_10

[14] International Maritime Organisation. IMO sets 2020 date for ships to comply with low sulphur fuel oil requirement [Online]. [Accessed 31.12.2019]. Available: http://www.imo.org/en/MediaCentre/PressBriefings/Pages/MEPC-702020sulphur.aspx

[15] Notteboom T. The impact of low sulphur fuel requirements in shipping on the competitiveness of roro shipping in Northern Europe. WMU Journal of Maritime Affairs 2011:10(1):63-95. https://doi.org/10.1007/s13437-010-0001-7 
[16] Olaniyi E. O., Prause G., Boyesen J. The Impact of SECA Regulations on Clean Shipping in the Baltic Sea Region. Trends and challenges in maritime energy management 2018:309-323. https://doi.org/10.1007/978-3-319-74576-3 22

[17] Atari S., Prause G. Risk assessment of emission abatement technologies for clean shipping. Proceedings of the International Conference on Reliability and Statistics in Transportation and Communication 2017:93-101. https://doi.org/10.1007/978-3-319-74454-4_9

[18] Prause G., et al. The Socio-Economic Impact of Green Shipping: A Holistic View from the Baltic Sea Region. Proceedings of the International Conference on Reliability and Statistics in Transportation and Communication 2018:615-624. https://doi.org/10.1007/978-3-030-12450-2_59

[19] Gerasimova V., Philipp R., Prause G. Service Design for Smart Supply Chains. Proceedings of the International Conference on Reliability and Statistics in Transportation and Communication 2021:[Forthcoming].

[20] Meyer C., Philipp R., Gerlitz L. Reinforcing Innovation and Competitiveness of SMEs by new Maritime Clustering Initiatives in South Baltic Sea Region. Proceedings of the International Conference on Reliability and Statistics in Transportation and Communication 2021:[Forthcoming].

[21] Philipp R., Gerlitz L., Moldabekova A. Small and Medium-Sized Seaports on the Digital Track: Tracing Digitalisation across the South Baltic Region by Innovative Auditing Procedures. Proceedings of the International Conference on Reliability and Statistics in Transportation and Communication 2020:351-362. https://doi.org/10.1007/978-3-03044610-9 35

[22] Philipp R. Digital Readiness Index Assessment towards Smart Port Development. Sustainability Management Forum 2020:28(1):49-60. https://doi.org/10.1007/s00550-020-00501-5

[23] Prause G., Atari S. On sustainable production networks for Industry 4.0. Entrepreneurship and Sustainability Issues 2017:4(4):421-431. https://doi.org/10.9770/jesi.2017.4.4(2)

[24] Olaniyi E. O., Atari S., Prause G. Maritime energy contracting for clean shipping. Transport and Telecommunication Journal 2018:19(1):31-44. https://doi.org/10.2478/ttj-2018-0004

[25] Olaniyi E. O., Gerlitz L. LNG Maritime energy contracting model. Entrepreneurship and Sustainability Issues 2019:7(1):574-594. http://doi.org/10.9770/jesi.2019.9.1(40)

[26] Osterwalder A., Pigneur Y. Business model creation. Amsterdam: Modderman Drukwerk, 2009.

[27] European Commission. Regulation (EU) No 1315/2013 of the European Parliament and of the Council of 11 December 2013 on Union guidelines for the development of the trans-European transport network and repealing Decision No 661/2010/EU Text with EEA relevance. Official Journal of the European Union 2013:L 348/1.

[28] European Commission. Regulation (EU) No 1316/2013 of the European Parliament and of the Council of 11 December 2013 establishing the Connecting Europe Facility, amending Regulation (EU) No 913/2010 and repealing Regulations (EC) No 680/2007 and (EC) No 67/2010 Text with EEA relevance. Official Journal of the European Union 2013:L348/129.

[29] Blumberga D., et al. Modelling of the Installed Capacity of Landfill Power Stations. Environmental and Climate Technologies 2009:3(3):19-26. https://doi.org/10.2478/v10145-009-0002-y

[30] Bengtsson S. K., Fridell E., Andersson K. E. Fuels for short sea shipping: A comparative assessment with focus on environmental impact. Proceedings of the Institution of Mechanical Engineers, Part M: Journal of Engineering for the Maritime Environment 2014:228(1):44-54. https://doi.org/10.1177/1475090213480349

[31] Pilicka I., Blumberga D., Romagnoli F. Life cycle assessment of biogas production from marine macroalgae: a Latvian scenario. Environmental and Climate technologies 2011:6(1):69-78. https://doi.org/10.2478/v10145-011-0010-6

[32] Blumberga A., Timma L., Blumberga D. System dynamic model for the accumulation of renewable electricity using power-to-gas and power-to-liquid concepts. Environmental and Climate Technologies 2015:16(1):54-68. https://doi.org/10.1515/rtuect-2015-0012

[33] Spoof-Tuomi K., Niemi S. Environmental and Economic Evaluation of Fuel Choices for Short Sea Shipping. Clean Technologies 2020:2(1):34-52. https://doi.org/10.3390/cleantechnol2010004

[34] Stenersen D., Thonstad O. GHG and $\mathrm{NO}_{\mathrm{x}}$ emissions from gas fuelled engines. Mapping, verification, reduction technologies. Trondheim: SINTEF Ocean AS, 2017.

[35] Spoof-Tuomi K., Niemi S. Emission reduction by biogas use in short sea shipping. Integrated energy solutions to smart and green shipping 2019:102-106.

[36] Henesey L., Lizneva Y., Philipp R., Meyer C., Gerlitz L. Improved load planning of roro vessels by adopting blockchain and internet-of-things. Proceedings of the 22nd International Conference on Harbor, Maritime and Multimodal Logistic Modeling \& Simulation (HMS 2020) 2020:58-65. https://doi.org/10.46354/i3m.2020.hms.009

[37] Moldabekova A., et al. Digital Technologies for Improving Logistics Performance of Countries. Transport and Telecommunication Journal 2021:[Forthcoming].

[38] Bleyl-Androschin J. W. Conservation first! The new integrated energy-contracting model to combine energy efficiency and renewable supply in large buildings and industry. Proceedings of European Council for an Energy Efficient Economy (ECEEE) 2011:1-14.

[39] Sorrell S. The economics of energy service contracts. Energy policy 2007:35(1):507-521. https://doi.org/10.1016/j.enpol.2005.12.009 
[40] GoLNG. Liquid Biogas Business Concept [Online]. [Accessed 31.12.2019]. Available: http:/www.golng.eu/files/Main/GoLNG_Blue_Corridor/Field\%20to\%20Ferry\%20business\%20model\%20concept_f inal.pdf

[41] Philipp R., Gerlitz L., Prause, G. Smart Contracts for Entrepreneurial Collaboration in Logistics Networks. Proceedings of the $11^{\text {th }}$ international scientific conference "New Challenges of Economic and Business Development - 2019. Incentives for Sustainable Economic Growth" 2019:652-663.

[42] Chuen D. L. K. Handbook of digital currency: Bitcoin, innovation, financial instruments, and big data. Singapore: Academic Press, 2015.

[43] Gallay O., et al. A peer-to-peer platform for decentralized logistics. In Proceedings of the Hamburg International Conference of Logistics (HICL) 2017:19-34. https://doi.org/10.15480/882.1473

[44] Liao D. Y., Wang X. Applications of Blockchain Technology to Logistics Management in Integrated Casinos and Entertainment. Informatics 2018:5(4):44. https://doi.org/10.3390/informatics5040044

[45] Manski S. Building the blockchain: the co-construction of a global commonwealth to move beyond the crises of global capitalism. Proceedings of the $12^{\text {th }}$ Annual California Graduate Student Conference 2016:1-29.

[46] Swan M. Blockchain: Blueprint for a new economy. Sebastopol: O'Reilly Media, Inc., 2015.

[47] Wu L. Blockchain Smart Contracts in Megacity Logistics. Pennsylvania: The Pennsylvania State University, 2018.

[48] Philipp R., Prause G., Gerlitz L. Blockchain and Smart Contracts for Entrepreneurial Collaboration in Maritime Supply Chains. Transport and Telecommunication Journal 2019:20(4):365-378. https://doi.org/10.2478/ttj-2019-0030

[49] L'Hermitte C., Wang W., Deakins E. Exploring the Physical Internet Concept to Improve Disaster Relief Operations. Proceedings of ISCRAM Asia Pacific 2018.

[50] Kouhizadeh M., Sarkis J. Blockchain Practices, Potentials, and Perspectives in Greening Supply Chains. Sustainability 2018:10(10):3652. https://doi.org/10.3390/su10103652

[51] García-Bañuelos L., et al. Optimized execution of business processes on blockchain. Proceedings of the International Conference on Business Process Management 2017:130-146. https://doi.org/10.1007/978-3-319-65000-5 8

[52] Kvale S. Doing interviews. London: Sage publications, 2008.

[53] Miles M. B., Huberman A. M. Qualitative data analysis: A sourcebook of new methods. Beverly Hills: Sage publications, 1984.

[54] Yin R. K. Case study research and applications: Design and methods. Newburry Park: Sage publications, 1989.

[55] Stopford M. Maritime Economics. New York: Routledge, 2009.

[56] Henesey L., Philipp R. Evaluating LNG Bunkering Automation Technology. World of Shipping Portugal: An International Research Conference on Maritime Affairs 2019:16-17.

[57] Langfeldt L., Pewe G. European Maritime Safety Agency (EMSA) - Study on Standards and Rules for bunkering of gas-fuelled Ships. Hamburg: Germanischer Lloyd, 2013.

[58] Norta A. Creation of smart-contracting collaborations for decentralized autonomous organizations. Proceedings of the International Conference on Business Informatics Research 2015:3-17. https://doi.org/10.1007/978-3-319-21915-8 1

[59] Stena Line. Supergreen mit methanol (Supergreen with methanol) [Online]. [Accessed 31.12.2019]. Available: https://www.stenaline.de/supergreen/treibstoff-der-zukunft (in German)

[60] IEA. Outlook for biogas and biomethane. World Energy Outlook Special Report. Paris: International Energy Agency, 2020.

[61] EIA - Independent Statistics and Analysis - U.S. Energy Information Administration. Natural Gas Prices [Online]. [Accessed 31.12.2019]. Available: https://www.eia.gov/dnav/ng/ng_pri_sum_dcu_nus_m.htm

[62] Finanzen.net. Dollarkurs (Dollar exchange rate) [Online]. [Accessed 31.12.2019]. Available: https://www.finanzen.net/devisen/dollarkurs/historisch (in German)

[63] Balland O. LNG - A cost-efficient fuel option? DNV.GL, 2014 [Online]. [Accessed 31.12.2019]. Available: https://www.sjofart.ax/sites/www.sjofart.ax/files/attachments/page/oceaneballand2014.pdf

[64] Madsen R. T. 144-Car Ferry LNG Fuel Conversion Feasibility Study. Life Cycle Cost Analysis. The Glosten Associates, 2011.

[65] Trading economics [Online]. [Accessed 31.12.2019]. Available: https://tradingeconomics.com/

[66] Ship \& Bunker - News and Intelligence for the Marine Fuels Industry [Online]. [Accessed 31.12.2019]. Available: https://shipandbunker.com/prices/emea/nwe/nl-rtm-rotterdam\#ULSFO

[67] Anderson M., Salo K., Fridell E. Particle-and gaseous emissions from an LNG powered ship. Environmental science \& technology 2015:49(20):12568-12575. https://doi.org/10.1021/acs.est.5b02678

[68] Burel F., Taccani R., Zuliani N. Improving sustainability of maritime transport through utilization of Liquefied Natural Gas (LNG) for propulsion. Energy 2013:57:412-420. https://doi.org/10.1016/j.energy.2013.05.002

[69] GoLNG. LNG infrastructure map [Online]. [Accessed 31.12.2019]. Available: http://www.golng.eu/en/bsr-lnginfrastructure-map/ 